\title{
Research on toy design based on the psychological and emotional needs of the elderly
}

\author{
Zhao Qian, Zuo Hongliang \\ (School of Art and Design, Henan University of Science and Technology, Luoyang, Henan 471023)
}

\begin{abstract}
This topic provides an overview of the research content in terms of background status, purpose, significance, etc., and analyzes the characteristics and needs of the elderly and the old toys on the current market. The literature research method and semantic difference method are used to further analyze the old toys. The causes and effects of user psychology and task design are studied to provide directions and ideas for toy design for the elderly in the future.
\end{abstract}

\section{Introduction}

With the development and progress of science and technology, China's social demographic structure is currently showing an aging state. Experts predict that by 2050, the number of elderly people in China will rank first in the world. Secondly, the advent of the information age has made products more intelligent, but the complexity of the interface and the diversification of functions have caused great emotional loss for the elderly. At the same time, the elderly have more free time after retirement and less family companionship, which undoubtedly enhances the degree of emotional loss of the elderly. In addition, foreign countries are older than China in toy design research, and are relatively mature in theoretical research and technical guidance. The research and development of elderly products in China are currently concentrated in health care and medical products. The design and research of elderly toys are still in the early stages, and there are few products available for the elderly to choose from on the market.

The methods used in this subject are the literature research method and the semantic difference method. By collecting and sorting out the literature, we can understand China's national conditions and explore the reasons from the perspective of design. The semantic difference method mainly converts the old people's perception of toys into perceptual evaluation data through perceptual evaluation, and then studies the relationship between the perception and design needs through quantitative analysis.

\section{Analysis of the characteristics of elderly toys and the elderly}

\subsection{Analysis of old toys}

Old toys, as the name suggests, are toys for the elderly. Because there are many kinds of toys involved in the elderly, they are mainly classified according to function during the research, which can be divided into: puzzle type, fitness type and entertainment type. After investigation, different types of old toys have different characteristics, as shown in Table 1:

Table 1. Classification of toys for the elderly.

\begin{tabular}{ll}
\hline Category & Function \\
\hline Puzzle type & $\begin{array}{l}\text { It has the effect of preventing Alzheimer's disease } \\
\text { Fitness type }\end{array}$ \\
$\begin{array}{l}\text { It has the functions of strengthening the body and slowing down the } \\
\text { degradation of physiological functions }\end{array}$ \\
$\begin{array}{l}\text { It increases the entertainment activities of the elderly and helps the } \\
\text { elderly pass their leisure time }\end{array}$ \\
\hline
\end{tabular}

In fact, the elderly play toys to help their physical and mental health, American medical experts have found that the elderly play toys can exercise their brains appropriately, the elderly who love toys play a $32 \%$ chance of suffering from dementia. In addition, psychologists also suggested that playing with toys can

Email: 1060064006@qq.com 
satisfy the spiritual needs of the elderly, while improving the quality of life of the elderly, it can also enrich the elderly's later life.

\subsection{Analysis of the characteristics of the elderly}

As the elderly grow older, their physiological functions are gradually declining, and due to retirement and other reasons, their roles in society will also be different. Therefore, there have been obvious changes in the physical and psychological aspects of the elderly.

With age, the elderly have degenerative changes in physiological functions. The main manifestations are: decreased activity, decreased hearing and hearing, and memory loss. The body's immune function declines and its resistance weakens. The internal environment's ability to balance is reduced, and its nutrient absorption capacity is poor.

Elderly people usually have the following psychological characteristics: Nostalgia, Seeking spiritual sustenance by recollecting the past. Loneliness and emptiness, longing for family companionship is the spiritual need of the elderly in the empty nest. Childlike innocence, the appearance of old age appears on the surface, but there is a shadow of the child in the behavior, accompanied by emotions such as strong curiosity and being asked to be cared for. In addition, the elderly often choose a regular lifestyle, focusing on health and rest. And the elderly have more time to retire after retirement, so the elderly have greater flexibility in their daily activities.

\section{Research process and results}

\subsection{Semantic difference method (SD method)}

Semantic difference method is an engineering research method, also known as "SD method". Through its research, it focuses on measuring the meaning of certain words or concepts understood by the testee, thus forming a semantic difference scale.

\subsection{Research process:}

By obtaining the collection of the research object's cognition or needs of the research product, collecting the sample data of the research content, analyzing and deconstructing the research object's needs and expectations of the research product, and then analyzing the reasonable and accurate user psychology and the cause and effect of the tasks design.

In the steps of the research process, first of all, you need to determine the research object according to the scope of the research content, and screen and determine the corresponding perceptual vocabulary through the semantic difference method (SD method), and the semantic difference scale and questionnaire design, then select the target group, fill in the questionnaire effectively, and finally collect the data in the questionnaire, and statistically analyze the data. The specific research steps are shown in Figure 1:

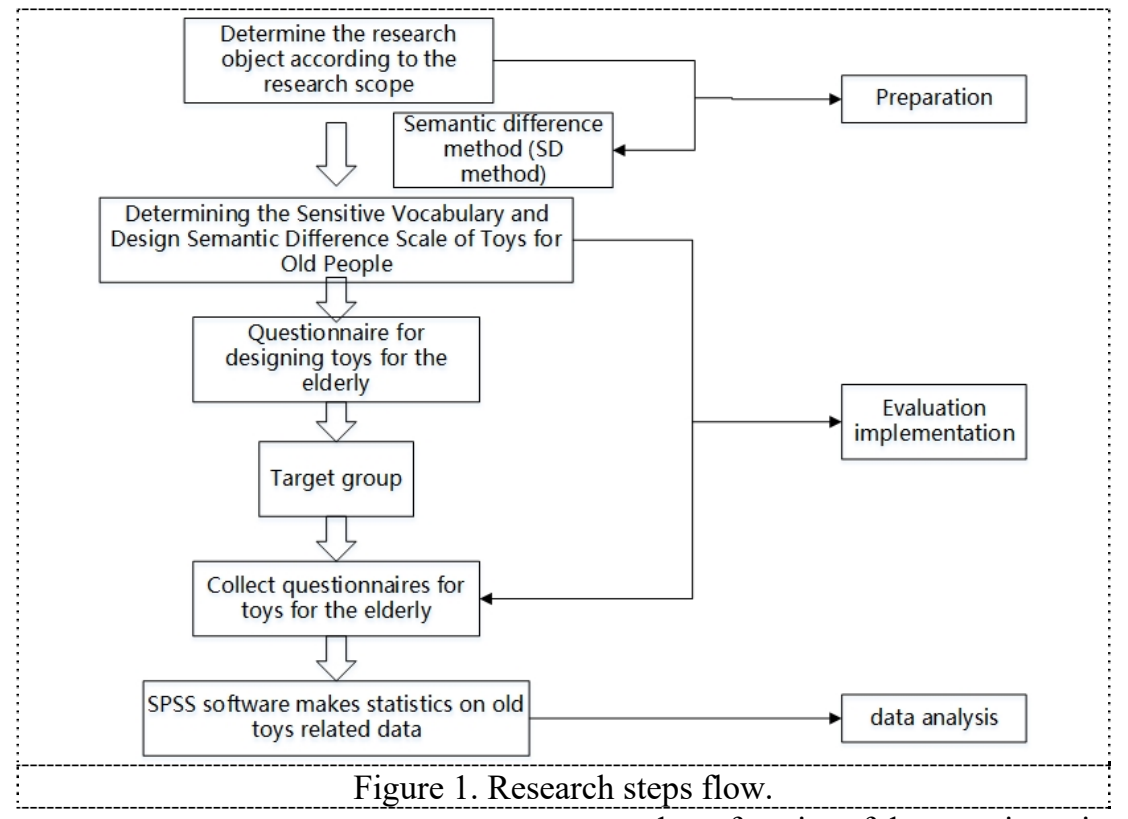

3.2.1 Design semantic difference scale. In this paper, 45 perceptual adjective vocabulary words are extracted from the old people's perception and feelings of old toys, and they are paired with antonyms. Each sample contains 15 pairs of perceptual adjective vocabulary words and 3 samples. Then, by adopting the seventh-order scale method in the form of a questionnaire, the testees were asked to rate and evaluate, and finally the 15 pairs of words with the highest scores were selected. The total number of copies of the questionnaire issued was 200, and the effective score of the returned questionnaire was 176.

3.2.2 Design questionnaire. Through careful design of the questionnaire, we can understand and grasp the situation of the elderly. The basic information in the questionnaire mainly includes: gender, education level, occupation or pre-retirement occupation, and income; the questions mainly involve: living conditions, entertainment activities, knowledge of old toys, considerations when purchasing, 
and The expectation and improvement measures of old toys. The total number of copies of the questionnaire sent was 300 , and the number of valid copies of the returned questionnaire was 274 .

\subsubsection{Statistics and analysis data. Reasonable statistics} and analysis of data are extremely important to the survey results. This paper uses EXCEL and SPSS software for statistical analysis.Sensitive adjective data analysis. Select the top 15 vocabulary words from the 45 perceptual adjective vocabularies and pair them with antonyms. From the statistics of the recovered data, it can be found that the test subjects pay more attention to the toys of the elderly in terms of appearance, interesting innovation, emotional interaction, difficulty, material technology and practicality.Data analysis of the questionnaire. Through the collection of questionnaires, statistics of the relevant data can be found from the statistics of the recovered data. Most elderly people 's education levels are concentrated in the junior and high school education, and the retirement income is concentrated in 1500-3000 yuan . In addition, $22.40 \%$ of the elderly's entertainment activities include square dance, and $18.67 \%$ of the elderly's entertainment activities include board games. It is not difficult to find that most elderly people are more engaged in fitness and educational entertainment activities. For older toys, more elderly people expect to focus on functions, fun, and safety, and improve on memory, reaction, and logical thinking.

\subsection{User psychology and task design}

3.3.1 Analysis of the causes and effects of user psychology. Design needs to serve users, so designers need to think from the perspective of users, and design products should start from the psychological characteristics of users. The result of the user's psychological effect can help the user to better understand the things he faces, and thus to live more effectively. To give a negative example: a user purchased a blue grip on an e-commerce website, but received an orange grip. The user's mental model is to get a blue grip, and the orange grip violates the user's psychology, and the final high probability result is a bad review. Through the analysis and research of perceptual adjectives and questionnaires, the following causes and effects of user psychology are obtained for the situation of the elderly, as shown in Figure 2:

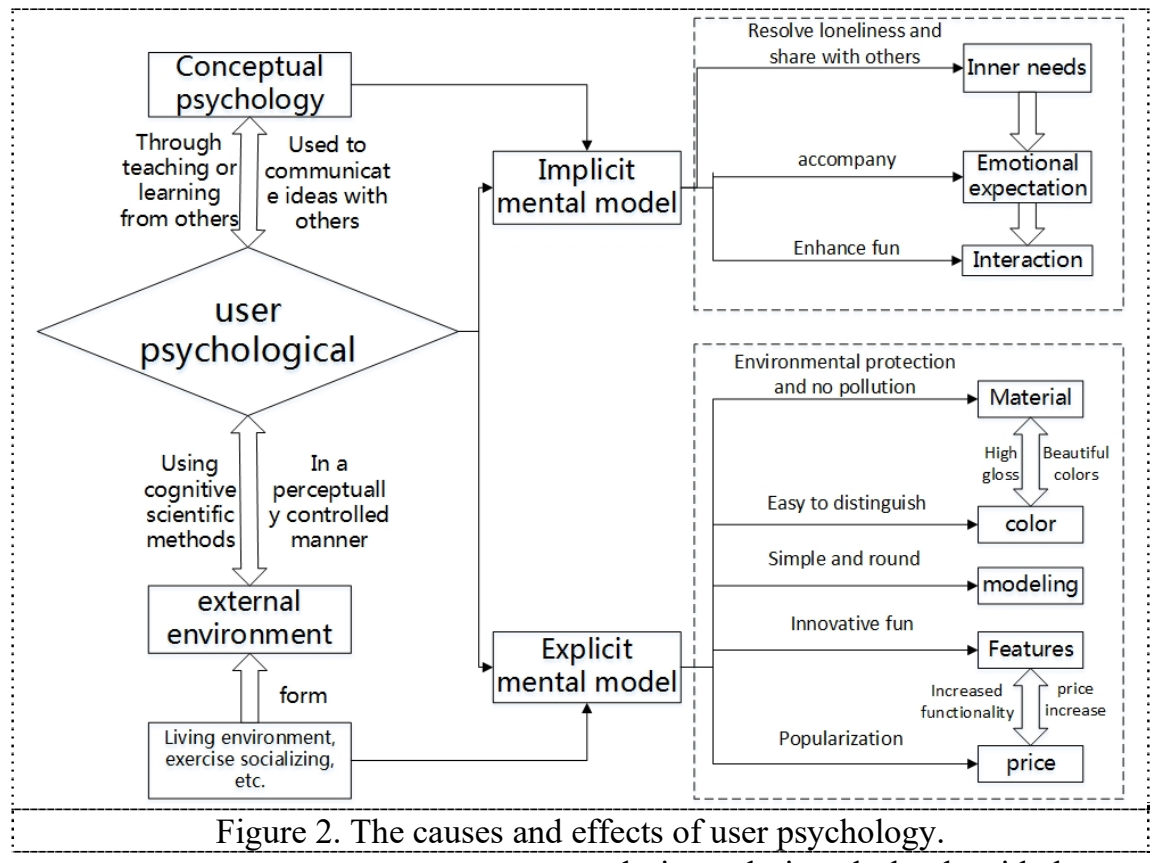

designer designed a kettle with the spout and the handle on

3.3.2 Analysis of the cause and effect of task design. Task design is that the designer designs the product according to the user's psychological causes and effects, such as the design of the product in terms of use mode, use function, use purpose, and use environment. The result of the role of task design can provide ideas and directions for the toy design of the elderly in the future. For example, the the same side without considering the user's functional requirements. This violates the user's psychology, and of course the designed product loses its original functionality. According to the user's psychology, the cause and effect of task design are obtained, as shown in Figure 3: 


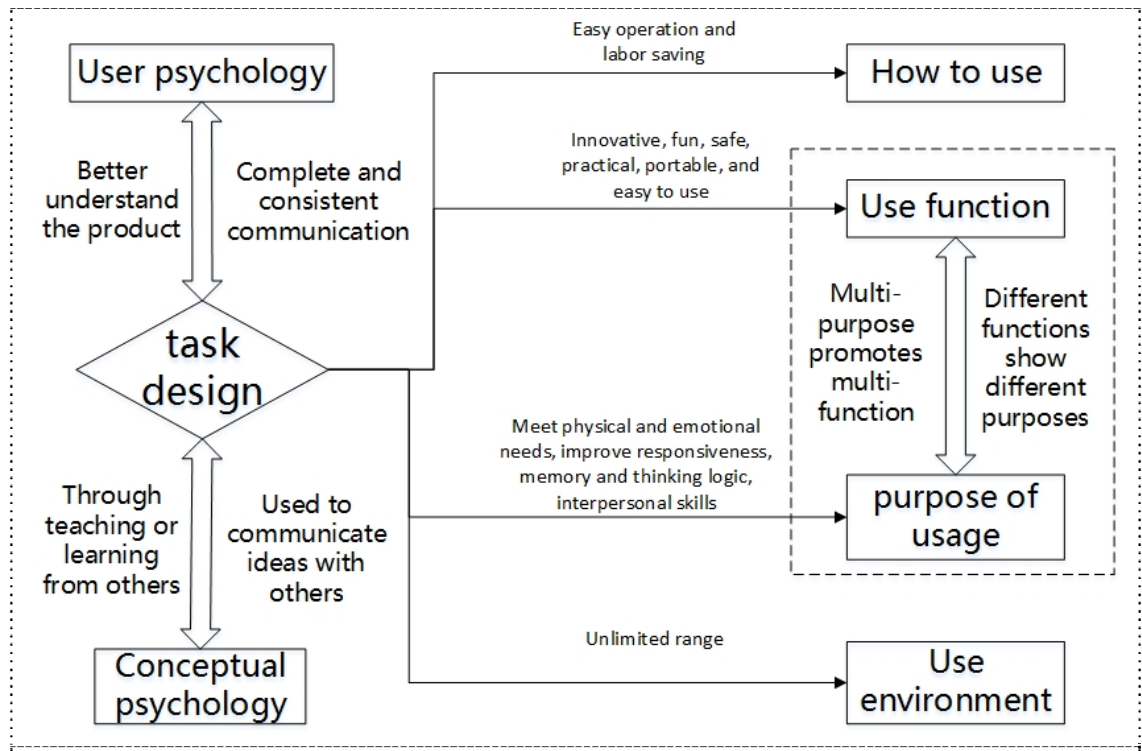

Figure 3. Causes and effects of task design

\section{Conclusion}

This article analyzes the psychological and emotional needs of the elderly, and uses the semantic difference method (SD method) to conduct qualitative and quantitative research on the toys of the elderly. Through the process of designing the semantic difference scale, designing the questionnaire, and using SPSS and EXCEL software to statistically analyze the results, the causes and effects of user psychology and task design are obtained, which provides directions and ideas for the toy design of the elderly in the future. In addition, the study of the article also has limitations and deficiencies. For example, there are only 274 valid questionnaires, and there is a problem of small sample size; at the same time, it also ignores the impact of differences between the elderly in different regions on the content of the study. I hope to make more in-depth research in the future when the research is more in-depth.

\section{References:}

1. Wang Lu. Research and design development of educational toys for the elderly [D]. Tianjin: Tianjin University of Science and Technology, 2014.

2. Liang Suqi. Analyzing toy design from the perspective of the emotional needs of the elderly [J]. Western Leather, 2016, 38 (14): 189-190.

3. Feng Wanqun. Research on the design of wearable body sign detection products for the elderly [D]. Jiangsu: China University of Mining and Technology, 2017.

4. Chen Zhuanqing. Research on toy design based on the emotional needs of the elderly [D]. Jiangsu: Jiangsu University, 2012.

5. Chen.A Study on Chinese elderly palm-toy design Development through E-FMS(Experience,Function,Material,Structure)sur vey[J].Industrial design,2015(04).

6. Zhang Gong. Analysis of the design direction and reference dimensions of toys for the elderly [J]. Mechanical Engineering and Automation, 2018, (1): 222-224.

7. Yang Rongman. Analysis on the physiological and psychological characteristics of the elderly and high-quality nursing $[\mathrm{J}]$. Chinese and Foreign Health Digest, 2011,08 (41): 305-306.

8. Hong-Ru.The Application Strategy of the Palm-toy Cultural Connotation for Chinese Elderly[J].Archives of Design Research,2015(04).

9. Li Yueen, Wang Zhenya, Xu Nan. Sensitive Engineering [M]. Beijing: Ocean Press, 2009: 77-79.

10. Luo Lixian, Hong Ling. Perceptual engineering design [M]. Beijing: Tsinghua University Press, 2015: 63-65.

11. Liu Qiao. Research on toy development and design based on the psychological needs of the elderly in China [D]. Tianjin: Tianjin University of Science and Technology, 2013.

12. Ren Jing. Research on the design of auxiliary products for the elderly based on ergonomics [D]. Shaanxi: Xi'an University of Architecture and Technology, 2013.

13. Zhang Tianhui. Research on the design of goal-oriented toys for the elderly $[\mathrm{J}]$. Literary Life · Literary Theory, 2016, (2): 46-46.

14. Ismail,Wesam Ibrahim.Suggested Multi-agent Model of Smart Home for Elderly[D]. Changsha: Hunan University, 2013.

15. Donald A Norman. Design Psychology 1-Daily Design [M]. Beijing: CITIC Press, 2015: 3. 